\title{
COMPARATIVE STUDY OF COMPRESSION TECHNIQUES FOR SYNTHETIC VIDEOS
}

\author{
Ayman Abdalla, Ahmad Mazhar, Mosa Salah, Sahar Khalaf
}

Faculty of Science \& I.T., Al-Zaytoonah University, Amman, Jordan

\begin{abstract}
We evaluate the performance of three state of the art video codecs on synthetic videos. The evaluation is based on both subjective and objective quality metrics. The subjective quality of the compressed video sequences is evaluated using the Double Stimulus Impairment Scale (DSIS) assessment metric while the Peak Signal-to-Noise Ratio (PSNR) is used for the objective evaluation. An extensive number of experiments are conducted to study the effect of frame rate and resolution on codecs' performance for synthetic videos. The evaluation results show that video codecs respond in different ways to frame rate and frame resolution change. H.264 shows superior capabilities compared to other codecs. Mean Opinion Score (MOS) results are shown for various bitrates, frame rates and frame resolutions.
\end{abstract}

\section{KEYWORDS}

CIF, DSIS, H.264, MOS, MPEG, QCIF, VP-8

\section{INTRODUCTION}

Synthetic videos are used by a wide variety of applications such as virtual reality tours, cartoons and games. Virtual reality tours require good compression techniques since they are often used in real time and require environment details to be shown. Online gaming has grown to become one of the largest entertainment sectors. More and more gamers upload videos of their games to video sharing websites or stream them live via online platforms such as Twitch TV. Therefore, video compression techniques are required to reduce the bitrate of gamers' video streams without compromising the quality. This study will investigate and compare the performance of the most popular standard video codecs on game and virtual reality videos. The compression of synthetic videos is a new research area. There are no specific studies on video codecs performance for synthetic videos.

In [1], a comparative study between H.264 and Motion JPEG2000 for high definition video coding was conducted. In [2], the performance of H.264, MPEG-4, H.263 and MPEG-2 were studied. The codecs were compared using (Peak Signal-to-Noise Ratio) PSNR and subjective measurements. In [3], an evaluation study of H.264 performance was conducted. The trade-off between coding efficiency and error resilience for network applications was considered, in addition to the end-to-end delay. In [4], an evaluation of perceptual visual quality under various settings and requirements was conducted. The subjective assessment tests were analyzed to study the influence of the different dimensions on the subjective evaluation. The considered dimensions are: encoder type, video content, bitrate, frame size and frame rate, where the only codecs used in the study were H.264 and H.263. In [5], a methodology was devised to evaluate the video

DOI : 10.5121/ijma.2014.6201 
The International Journal of Multimedia \& Its Applications (IJMA) Vol.6, No.2, April 2014

perceived quality when watching high motion videos. The video sequences were chosen from some football matches with different physical quality metrics. CIF and QCIF frame resolutions were used to study the relationship between frame rate and quantization and their impact on the perceived quality. In [6], a new objective quality metric for video sequences was proposed. The proposed metric was based on estimating the MOS of compressed sequences using MSE, spatial video content and skin information. The idea focused on the factors that attract viewer attention. In [7], a review of video quality measurement techniques was performed. Both subjective and objective quality metrics were discussed, in addition to a hybrid metric. In [8], an evaluation study of present video compression techniques effects on the perceptual quality of video coding was presented. In [9], a study of the rating scale in subjective testing methodology was presented. The study focused on the importance of the rating scale elements and their effect on the subjective evaluation results. In [10], a wide comparative study between H.264 and MPEG-4 was conducted. Most technical differences between the two codecs were presented. The results showed the superiority of codec H.264 over MPEG-4.

Three types of video codecs are selected for the study in this paper; H.264, MPEG-4 and VP8. The sequences used to perform this comparison study were captured from the "World of Warcraft" game. This game is considered one of the most popular online games. It contains a wide range of features and visual specifications. The sequences were captured using a software tool called FRAPS [11]. The goal of this paper is to study the most possible frame rates and resolutions over the common bitrate values, and show the effects of this variation on video codecs performance for synthetic sequences. We also discuss the degree of codec sensitivity to this variation. The study addressed subjective and objective measurements for a comprehensive study.

\section{METHODOLOGY OF THE STUDY}

Small frame resolutions with QCIF and CIF sizes were selected for making comparisons in this study. Three different frame rates; 15 frames per second (fps), $25 \mathrm{fps}$ and $30 \mathrm{fps}$, were selected. Videos were compressed at 256, 512 and $1024 \mathrm{kbps}$. These bitrates were selected to suit the average internet speeds available in most countries. According to [12-14], the selected bitrates match the lowest available internet speed in the UK and in Jordan where the study was conducted. The compression experiments are applied over diverse types of captured video sequences in order to provide different scene contents. The study considered scenes that include sequences with high-texture high-motion, high-texture low-motion, low-texture high-motion and low-texture low-motion contents. Compression was performed using the FFmpeg software [15]. The process used in this study to evaluate the codecs performance includes both subjective and objective metrics. Peak signal to noise ratio (PSNR) was used as the objective metric since it is widely used by the video processing community. It is easy to implement and fast to compute [7]. The DSIS variant II, which is recommended by the ITU [16], was used as the subjective quality metric.

Four ten-second test video sequences were viewed by fifteen viewers, where different spatial and temporal resolutions were considered. The original video sequence is displayed to the viewer first; and then the compressed video sequence is displayed. The viewers cannot score before this process is repeated. After that, the viewers give their impression on the compression quality, compared to the original sequences, using a five-level quality scale. All viewers are B.Sc. students with good communication skills. The experiments were conducted in a computer lab using 19" Dell LCD monitors. The lab's windows were covered with gray curtains and white lights were used. All students used the same lab and monitor specifications. For the subjective evaluation, all video sequences were displayed at the same resolution and frame rate. The frame size $4 \mathrm{CIF}$ and frame rate $30 \mathrm{fps}$ were chosen for all tests. The lower resolutions were upsampled to $4 \mathrm{CIF}$ and lower frame rates were repeated to become $30 \mathrm{fps}$. The process of upsampling and 
The International Journal of Multimedia \& Its Applications (IJMA) Vol.6, No.2, April 2014

frame repetition was conducted using the H.264/AVC 6-tap half-sample interpolation filter and frame repeat [4].

\section{OBJECTIVE EXPERIMENTAL RESULTS}

The comparative study with results analysis is presented in this section. The effect of frame rates and bitrates with CIF and QCIF resolutions are also illustrated. As shown in Table 1, H.264 outperformed MPEG-4 and VP-8 at $1024 \mathrm{kbps}$ CIF resolution. H.264 PSNR was more than 2 decibels $(\mathrm{dB})$ higher than the PSNR of the other two codecs and the PSNR difference decreased as the bitrate decreased. H.264 still performed better than other codecs at $512 \mathrm{kbps}$, and the difference decreased as the frame rate decreased at the same bitrate. MPEG-4 performed slightly better with $256 \mathrm{kbps}$ than with the other frame rates. At QCIF, H.264 significantly outperformed other codecs at higher bitrates. PSNR was around $4 \mathrm{~dB}$ better than VP- 8 and $6 \mathrm{~dB}$ than MPEG-4 at $512 \mathrm{kbps}$.

Table 1: HTHM PSNR

\begin{tabular}{|c|c|c|c|c|c|c|c|}
\hline \multirow{3}{*}{$\begin{array}{c}\text { Bit } \\
\text { Rate } \\
\text { (kbps) }\end{array}$} & \multirow{3}{*}{$\begin{array}{c}\text { Frame } \\
\text { Rate } \\
\text { (fps) }\end{array}$} & \multicolumn{6}{|c|}{ PSNR (dB) } \\
\hline & & \multicolumn{2}{|c|}{ H.264 } & \multicolumn{2}{|c|}{ MPEG-4 } & \multicolumn{2}{|c|}{ VP-8 } \\
\hline & & QCIF & CIF & QCIF & CIF & QCIF & CIF \\
\hline \multirow{3}{*}{256} & 15 & 41.95 & 34.01 & 41.69 & 35.39 & 39.42 & 32.17 \\
\hline & 25 & 41.47 & 33.63 & 42.71 & 35.05 & 39.28 & 33.97 \\
\hline & 30 & 41.32 & 33.64 & 43.37 & 35.02 & 39.11 & 33.84 \\
\hline \multirow{3}{*}{512} & 15 & 50.1 & 37.76 & 43.31 & 37.55 & 46.34 & 37.38 \\
\hline & 25 & 49.5 & 37.42 & 43.5 & 37.09 & 45.85 & 37.26 \\
\hline & 30 & 49.38 & 37.52 & 43.6 & 37.06 & 45.65 & 37.15 \\
\hline \multirow{3}{*}{1024} & 15 & 64.89 & 43.33 & 43.32 & 40.68 & 57.47 & 41.17 \\
\hline & 25 & 64.15 & 43.05 & 43.54 & 40.35 & 57.56 & 40.88 \\
\hline & 30 & 63.38 & 43.27 & 43.61 & 40.13 & 57.63 & 40.61 \\
\hline
\end{tabular}

The results in Table 2 show the average PSNR for the HTLM sequences of the three codecs. H.264 shows, on average, a $3 \mathrm{~dB}$ better performance than MPEG-4 and VP- 8 at $1024 \mathrm{kbps}$. The difference between H.264 and VP-8 decreased at $512 \mathrm{kbps}$ where it was less than $1 \mathrm{~dB}$ on average. The VP-8 codec showed a slightly better performance than H.264 at $256 \mathrm{kbps}$. However, VP-8 outperformed other codecs at lower bitrates. The VP-8 codec showed a slightly better performance than H.264 at the low bitrate.

Table 2: HTLM PSNR

\begin{tabular}{|c|c|c|c|c|c|c|c|}
\hline \multirow{3}{*}{$\begin{array}{c}\text { Bit } \\
\text { Rate } \\
\text { (kbps) }\end{array}$} & \multirow{3}{*}{$\begin{array}{l}\text { Frame } \\
\text { Rate } \\
\text { (fps) }\end{array}$} & \multicolumn{6}{|c|}{ PSNR (dB) } \\
\hline & & \multicolumn{2}{|c|}{ H.264 } & \multicolumn{2}{|c|}{ MPEG-4 } & \multicolumn{2}{|c|}{ VP-8 } \\
\hline & & $\overline{\text { QCIF }}$ & CIF & $\overline{\text { QCIF }}$ & CIF & $\overline{\text { QCIF }}$ & CIF \\
\hline \multirow{3}{*}{256} & 15 & 52.07 & 42.6 & 43.64 & 39.31 & 47.18 & 43.01 \\
\hline & 25 & 49.73 & 41.56 & 43.85 & 39.65 & 45.54 & 42.18 \\
\hline & 30 & 48.86 & 40.95 & 43.9 & 39.63 & 45.11 & 41.11 \\
\hline \multirow{3}{*}{512} & 15 & 61.53 & 47.96 & 43.64 & 42.52 & 56.39 & 46.38 \\
\hline & 25 & 58.27 & 46.76 & 43.85 & 42.44 & 52.18 & 45.95 \\
\hline & 30 & 56.96 & 46.09 & 43.9 & 42.37 & 51.23 & 45.42 \\
\hline \multirow{3}{*}{1024} & 15 & 62.83 & 55.5 & 43.65 & 44.6 & 56.58 & 52.67 \\
\hline & 25 & 62.31 & 53.26 & 43.86 & 44.77 & 56.33 & 50.06 \\
\hline & 30 & 62.05 & 52.33 & 43.91 & 44.79 & 56.19 & 49.16 \\
\hline
\end{tabular}


The International Journal of Multimedia \& Its Applications (IJMA) Vol.6, No.2, April 2014

The QCIF results in Table 2 show that H.264 significantly outperformed other codecs, as it gave over $6 \mathrm{~dB}$ more than MPEG-4 and over $4 \mathrm{~dB}$ better than VP-8 at $256 \mathrm{kbps}$. The average PSNR values for LTHM video sequences are shown in Table 3. All codecs showed better PSNR when increasing the bitrate, where they decreased when increasing the frame rate. H.264 showed better PSNR than other codecs at higher bitrates. The QCIF results show that MPEG-4 capability is close to H.264 at a lower bitrate, where the difference was less than $1 \mathrm{~dB}$ on average. Conversely, VP-8 gave the lowest PSNR at 256 kbps. At higher bitrates, H.264 showed an increase in PSNR with an increase in the difference from other codecs.

Table 3: LTHM PSNR

\begin{tabular}{|c|c|c|c|c|c|c|c|}
\hline \multirow{3}{*}{$\begin{array}{c}\text { Bit } \\
\text { Rate } \\
\text { (kbps) }\end{array}$} & \multirow{3}{*}{$\begin{array}{c}\text { Frame } \\
\text { Rate } \\
\text { (fps) }\end{array}$} & \multicolumn{6}{|c|}{ PSNR (dB) } \\
\hline & & \multicolumn{2}{|c|}{ H.264 } & \multicolumn{2}{|c|}{ MPEG-4 } & \multicolumn{2}{|c|}{ VP-8 } \\
\hline & & $\overline{\text { QCIF }}$ & CIF & $\overline{\text { QCIF }}$ & CIF & $\overline{\text { QCIF }}$ & CIF \\
\hline \multirow{3}{*}{256} & 15 & 42.2 & 37.57 & 41.2 & $\overline{37.04}$ & 39.96 & 38.42 \\
\hline & 25 & 41.96 & 37.07 & 41.07 & 36.87 & 39.85 & 37.96 \\
\hline & 30 & 41.9 & 36.85 & 41.58 & 37.38 & 39.65 & 37.63 \\
\hline \multirow{3}{*}{512} & 15 & 46.96 & $\overline{41.49}$ & 43.81 & 40.12 & 44.47 & 41.63 \\
\hline & 25 & 46.87 & 41.17 & 43.91 & 39.45 & 44.16 & 41.35 \\
\hline & 30 & 46.85 & 41.05 & 43.96 & 39.48 & 43.89 & 41.19 \\
\hline \multirow{3}{*}{1024} & 15 & 52.66 & 45.56 & 43.82 & 43.76 & 44.09 & 44.76 \\
\hline & 25 & 52.78 & 45.37 & 43.92 & 42.91 & 43.88 & 44.47 \\
\hline & 30 & 52.8 & 45.31 & 43.96 & 42.94 & 43.75 & 44.29 \\
\hline
\end{tabular}

Table 4 shows the average PSNR of LTLM. As shown in the table, the H.264 codec at CIF resolution outperformed other codecs at the higher bitrates. H.264 performed on average $2 \mathrm{~dB}$ better than VP-8 and was approximately $14 \mathrm{~dB}$ better than MPEG-4 at 1024 kbps. For QCIF resolution, H.264 showed better performance than other codecs for all sequences. It was about 13 $\mathrm{dB}$ better than MPEG-4 and about $1 \mathrm{~dB}$ better, on average, compared to VP- 8 at $256 \mathrm{kbps}$. However, this difference clearly decreased as the frame rate increased.

Table 4: LTLM PSNR

\begin{tabular}{|c|c|c|c|c|c|c|c|}
\hline \multirow{3}{*}{$\begin{array}{c}\text { Bit } \\
\text { Rate } \\
\text { (kbps) }\end{array}$} & \multirow{3}{*}{$\begin{array}{l}\text { Frame } \\
\text { Rate } \\
\text { (fps) }\end{array}$} & \multicolumn{6}{|c|}{ PSNR (dB) } \\
\hline & & \multicolumn{2}{|c|}{ H.264 } & \multicolumn{2}{|c|}{ MPEG-4 } & \multicolumn{2}{|c|}{ VP-8 } \\
\hline & & QCIF & CIF & $\overline{\text { QCIF }}$ & CIF & QCIF & CIF \\
\hline \multirow{3}{*}{256} & 15 & 60.82 & 47.95 & 44.08 & 42.5 & 57.89 & 48.7 \\
\hline & 25 & 57.32 & 44.36 & 44.15 & 40.44 & 55.73 & 44.51 \\
\hline & 30 & 54.72 & 42.84 & 44.172 & 39.88 & 54.19 & 43.18 \\
\hline \multirow{3}{*}{512} & 15 & 63.54 & 55.55 & 44.08 & 44.37 & 61.42 & 53.64 \\
\hline & 25 & 62.84 & 50.78 & 44.15 & 44.42 & 60.68 & 49.47 \\
\hline & 30 & 61.87 & 48.93 & 44.172 & 43.42 & 60.17 & 48.21 \\
\hline \multirow{3}{*}{1024} & 15 & 64 & 62.44 & 44.08 & 44.38 & 61.78 & 60.07 \\
\hline & 25 & 64.06 & 59.66 & 44.15 & 44.43 & 60.58 & 57.81 \\
\hline & 30 & 63.83 & 57.42 & 44.173 & 44.44 & 58.62 & 55.36 \\
\hline
\end{tabular}

\section{SUBJECTIVE EXPERIMENTAL RESULTS}

The average DSIS of HTHM and CIF resolution is shown in Table 5. The results show that the users preferred the sequences compressed by the H.264 codec to those compressed using MPEG4 and VP-8. The table also shows the average DSIS for this type of sequences. The results show that H.264 gave better performance than other codecs, while the performance of VP- 8 came in second. However, results illustrate that all codecs achieved around 3 out of 5 on average in this 
The International Journal of Multimedia \& Its Applications (IJMA) Vol.6, No.2, April 2014

subjective evaluation. The reason for this result is the complexity of this type of sequences and the compression difficulty that the codecs encountered.

The subjective evaluation results of HTLM are shown in Table 6. At CIF resolution, the H.264 codec produced the highest satisfaction with all bitrates. VP-8 was better than MPEG-4 with all different bitrates. The difference between codecs increased as the bitrate increased and H.264 started getting better results. However, the QCIF frame size showed that VP-8 and H.264 had similar evaluation results at $256 \mathrm{kbps}$, where both codecs were better than MPEG-4. When increasing the bitrate, H.264 started to perform better than VP-8. Results show that the difference increased as the bitrate increased.

Table 5: HTHM MOS Results

\begin{tabular}{|c|c|c|c|c|c|c|}
\hline \multirow{2}{*}{ Codec } & \multicolumn{6}{|c|}{ Bit Rate } \\
\cline { 2 - 7 } & \multicolumn{2}{|c|}{$\mathbf{2 5 6}$} & \multicolumn{2}{|c|}{$\mathbf{5 1 2}$} & \multicolumn{2}{c|}{$\mathbf{1 0 2 4}$} \\
\cline { 2 - 7 } & CIF & QCIF & CIF & QCIF & CIF & QCIF \\
\hline H.264 & 4.2 & 3 & 4.33 & 3.33 & 4.46 & 3.53 \\
\hline MPEG-4 & 3.33 & 2.4 & 3.66 & 2.4 & 3.8 & 3 \\
\hline VP-8 & 3.73 & 2.6 & 3.93 & 3.06 & 4.26 & 3.46 \\
\hline
\end{tabular}

Table 6: HTLM MOS Results

\begin{tabular}{|c|c|c|c|c|c|c|}
\hline \multirow{2}{*}{ Codec } & \multicolumn{6}{|c|}{ Bit Rate } \\
\cline { 2 - 7 } & \multicolumn{2}{|c|}{$\mathbf{2 5 6}$} & \multicolumn{2}{|c|}{$\mathbf{5 1 2}$} & \multicolumn{2}{|c|}{$\mathbf{1 0 2 4}$} \\
\cline { 2 - 7 } & CIF & QCIF & CIF & QCIF & CIF & QCIF \\
\hline H.264 & 3.73 & 3.13 & 4.13 & 3.26 & 4.46 & 3.66 \\
\hline MPEG-4 & 3.2 & 2.8 & 3.73 & 2.93 & 3.66 & 3 \\
\hline VP-8 & 3.66 & 3.13 & 4 & 3.2 & 4.2 & 3.4 \\
\hline
\end{tabular}

Table 7: LTHM MOS Results

\begin{tabular}{|c|c|c|c|c|c|c|}
\hline \multirow{2}{*}{ Codec } & \multicolumn{9}{|c|}{ Bit Rate } \\
\cline { 2 - 7 } & \multicolumn{2}{|c|}{$\mathbf{2 5 6}$} & \multicolumn{2}{|c|}{$\mathbf{5 1 2}$} & \multicolumn{2}{|c|}{$\mathbf{1 0 2 4}$} \\
\cline { 2 - 7 } & CIF & QCIF & CIF & QCIF & CIF & QCIF \\
\hline H.264 & 4.33 & 3.33 & 4.2 & 3.4 & 4.4 & 3.66 \\
\hline MPEG-4 & 3.93 & 3.06 & 4.13 & 3.06 & 4.06 & 3.33 \\
\hline VP-8 & 4.13 & 3.33 & 4.13 & 3.33 & 4.33 & 3.46 \\
\hline
\end{tabular}

Table 8: LTLM MOS Results

\begin{tabular}{|c|c|c|c|c|c|c|}
\hline \multirow{2}{*}{ Codec } & \multicolumn{6}{|c|}{ Bit Rate } \\
\cline { 2 - 7 } & \multicolumn{2}{|c|}{$\mathbf{2 5 6}$} & \multicolumn{2}{|c|}{$\mathbf{5 1 2}$} & \multicolumn{2}{|c|}{$\mathbf{1 0 2 4}$} \\
\cline { 2 - 7 } & CIF & QCIF & CIF & QCIF & CIF & QCIF \\
\hline H.264 & 4.2 & 3.33 & 4.33 & 3.53 & 4.66 & 4 \\
\hline MPEG-4 & 3.53 & 3.13 & 4.13 & 2.93 & 4.06 & 3.13 \\
\hline VP-8 & 4.13 & 3.33 & 4.26 & 3.46 & 4.6 & 3.73 \\
\hline
\end{tabular}

Table 7 illustrates the average DSIS of LTHM sequences. All codecs got high averages in the CIF evaluation results. However, it is clear that the H.264 codec got better subjective evaluation than other codecs at all bitrates. Results of H.264 and VP-8 for QCIF were similar at a lower bitrate and close at higher rate. The difference slightly increased in favor of H.264 as the bitrate rate increased. Table 8 demonstrates the LTLM average DSIS. H.264 and VP-8 DSIS values for CIF 
The International Journal of Multimedia \& Its Applications (IJMA) Vol.6, No.2, April 2014

were almost equal with a little preference for H.264. DSIS of both codecs were better than MPEG-4 at all bitrates rates and frame rates. At 256 kbps QCIF resolution, both H.264 and VP-8 showed comparable results, whereas both codecs were slightly better than MPEG-4. At higher bitrates rates, H.264 started performing better than VP-8 with a small difference at $512 \mathrm{kbps}$ and increased at $1024 \mathrm{kbps}$.

\section{STATISTICAL STUdy OF SUbJECTIVE RESUlTS}

The PSNR and MOS results of codec comparison were shown in the preceding section. The descriptive statistical study in the section showed these simple observations: First, H.264 codec outperformed both MPEG-4 and VP-8. Second, the type of video sequence and frame rate affected MOS, where the higher the motion and texture video sequences are the higher the MOS becomes.

For completeness of studying the influence of different dimensions on MOS, this section considers a wider scope of subjective video quality assessment analysis. The evaluating of perceptual visual quality under different requirements has been performed including five distinctive dimensions: encoder type, video type (content), bitrate, frame rate and frame size.

The obtained subjective results have been statistically analyzed. The influence of the different dimensions on MOS has been illustrated. The Analysis of Variance (ANOVA) is used to conduct this evaluation. ANOVA is a statistical technical method that can be used to compare the means of two or more groups under certain assumptions. There are several types of ANOVA found in the literature for the purpose of comparison such as the one-way ANOVA, two-way ANOVA, factorial analysis and other types. Refer to [17] for details of ANOVA. To test the effect of video type (VT), Codec Type (CT), frame per second (FpS) and bitrate (BR) on MOS, three-way ANOVA was adopted in this analysis.

The analysis focuses on capturing any difference in MOS mean under the above potential factors. The model that will be tested is as in the following equation:

$$
\mathrm{MOS}=\mathrm{BR}+\mathrm{FpS}+\mathrm{VT}+\varepsilon
$$

In the above model, it should be noted that the error is assumed normally and identically distributed with mean 0 and variance $\sigma^{2}$. Before applying the analysis, three assumptions for ANOVA should be validated:

1. Normality assumption: We resort to large sample theory to justify the normality of our sample since the sample size is over 30 .

2. Independency: The independency assumption is satisfied since each DSIS result is not affected by the others.

3. Homoscedasticity (equality of variances): Levene's Test of Equality of Error Variances is conducted and the results are shown in Table 9. Results show that no major violation of this assumption is observed.

In order to perform the statistical study of the three-way ANOVA, a set of treatments is determined to study the influence of different dimensions on MOS where the variables are Codec Type (CT), Video Type (VT) and Bitrate (BR).

Table 9: Test of Equality of Error Variances

\begin{tabular}{|c|c|c|c|}
\hline F & df1 & df2 & Sig. \\
\hline 0.902 & 11 & 36 & 0.548 \\
\hline
\end{tabular}


The International Journal of Multimedia \& Its Applications (IJMA) Vol.6, No.2, April 2014

Table 10: Three-Way ANOVA on MOS of QCIF

\begin{tabular}{|c|c|c|c|c|c|}
\hline Dimensions & Sum of Squares & Degrees of Freedom & Mean Square & F statistic & p-value \\
\hline CT & 1.819 & 2 & 0.909 & 15.497 & 0.0000 \\
\hline VT & 1.809 & 3 & 0.603 & 10.276 & 0.0002 \\
\hline BR & 0.548 & 2 & 0.274 & 4.671 & 0.018 \\
\hline
\end{tabular}

The analysis results of QCIF are illustrated in Table 10. As shown in the table, p-values are small $(p \leq 0.05)$. The smallness of $p$-values indicates that the influence of the dimensions is substantial and all have significant effect on MOS. In addition, the magnitudes of these dimensions are indicators of the strength of the influence on the MOS. The CT affects MOS the most followed by VT. The least impact is of BR.

For codecs performance evaluation, the pairwise comparison results are shown in Table 11 and Table 12. The results show that the (p-values) are significant for CT and substantially affect MOS between H.264 and MPEG-4, and highly affected it between VP-8 and MPEG-4. Furthermore, positive mean difference value where (I is H.264 or VP-8) means that the advantage of the significance is for the codec represented by (I). On the contrary, negative value indicates that the advantage is for the codec that is represented by $(\mathrm{J})$.

MOS was not significantly affected when switching between VP-8 and H.264, but MOS was noticeably affected and viewers could easily sense that some changes happened when compression was used. This implies that MOS is severely affected by CT when comparing H.264 or VP-8 with MPEG-4 (p-values < 0.05). When comparing H.264 to VP-8, the experiments showed that the results are insignificant ( $\mathrm{p}$-values $>0.05$ ). However, mean difference results showed that H.264 insignificantly outperforms VP-8 as the positive value was obtained when $(\mathrm{I}=$ H.264) but with (p-value $>0.05$ ). The detailed pairwise comparison results of QCIF statistical study are shown in Table 12.

Table 11: Pairwise of Video Codecs of CIF

\begin{tabular}{|c|c|c|c|}
\hline \multirow{2}{*}{ (I) CT } & (J) CT & $\begin{array}{c}\text { Mean Difference } \\
\text { (I-J) }\end{array}$ & p-value \\
\hline \multirow{2}{*}{ H264 } & MPEG-4 & 0.532 & 0.000 \\
\cline { 2 - 4 } & VP-8 & 0.145 & 0.154 \\
\hline \multirow{2}{*}{ MPEG-4 } & H264 & -0.532 & 0.000 \\
\cline { 2 - 4 } & VP-8 & -0.387 & 0.001 \\
\hline \multirow{2}{*}{ VP-8 } & H264 & -0.145 & 0.154 \\
\cline { 2 - 4 } & MPEG-4 & 0.387 & 0.001 \\
\hline
\end{tabular}

Table 12: Pairwise of Video Codecs of QCIF

\begin{tabular}{|c|c|c|c|}
\hline \multirow{2}{*}{ (I) CT } & (J) CT & $\begin{array}{c}\text { Mean Difference } \\
\text { (I-J) }\end{array}$ & p-value \\
\hline \multirow{2}{*}{ H264 } & MPEG-4 & 0.496 & 0.000 \\
\cline { 2 - 4 } & VP-8 & 0.173 & 0.007 \\
\hline \multirow{2}{*}{ MPEG-4 } & H264 & -0.496 & 0.000 \\
\cline { 2 - 4 } & VP-8 & -0.323 & 0.000 \\
\hline \multirow{2}{*}{ VP-8 } & H264 & -0.173 & 0.007 \\
\cline { 2 - 4 } & MPEG-4 & 0.323 & 0.000 \\
\hline
\end{tabular}


The International Journal of Multimedia \& Its Applications (IJMA) Vol.6, No.2, April 2014

Table 13: Three-Way ANOVA on MOS of CIF

\begin{tabular}{|c|c|c|c|c|c|}
\hline Dimensions & Sum of Squares & Degrees of Freedom & Mean Square & F statistic & p-value \\
\hline CT & 1.520 & 2 & 0.760 & 35.067 & 0.000 \\
\hline VT & 0.625 & 3 & 0.208 & 9.610 & 0.000 \\
\hline BR & 0.805 & 2 & 0.402 & 18.572 & 0.000 \\
\hline
\end{tabular}

The results of the three-way ANOVA of CIF format are illustrated in Table 13. As shown in the table, all dimensions are significant, where p-values are almost zero. It can be noticed in the sixth column that $(\mathrm{p} \leq 0.05)$. This indicates that the influence of the dimensions is substantially significant for CT, VT and BR on MOS. In addition, the magnitudes of BR are less than the BR magnitude of QCIF. Therefore, this is considered as an indicator of the smaller strength of influence of BR on MOS compared to the most BR influence in QCIF. In CIF format, the effect of CT, VT and BR on MOS is comparable.

The Pairwise comparison results of CIF are shown in Table 11. The results show that (p-values) are significant of CT and are substantially affects the MOS between H.264 and MPEG-4, MOS is also highly affected between VP-8 and MPEG-4. Mean Difference has either positive or negative values, where (I is H.264 and J is VP-8). Where positive results mean that the advantage of the significance is for the codec represented by (I), and negative value indicates that the advantage is for the codec that is represented by $(\mathrm{J})$, result implies that MOS is severely affected by CT when comparing H.264 or VP-8 with MPEG-4.

When comparing H.264 to VP-8, the results showed a different case than in QCIF. It is clear that $(p \leq 0.05)$ which means it is significant and MOS is severely affected by changing from one codec to the other. In addition, Mean Difference results show that H.264 severely outperformed VP-8 since the positive value was obtained when $(\mathrm{I}=\mathrm{H} .264)$. As a conclusion, we can claim that H.264 outperforms both codecs at CIF and QCIF resolutions with considerable significance of QCIF.

\section{CONCLUSIONS}

The effects of frame rate and resolution on the most popular video codecs performance and perceptual quality were evaluated. The study was conducted using PSNR, the most popular objective metric, and extensive subjective viewing tests using DSIS II. The frame rates and resolutions were tested at low bitrates currently available in typical uplink streaming. The results led to new interesting perceptions of the online video gaming compression techniques. First, the H.264 video coding technique showed high performance on synthetic video coding. Second, perceptual quality was affected significantly by VT and CT more than the effect by BR at the QCIF resolution. However, it was insignificantly affected at higher resolutions such as CIF. Third, the higher frame sizes with lower frame rates were more acceptable by observers, especially for high texture sequences. Fourth, VP-8 showed comparable compression capability which makes it a high competitor with H.264 and MPEG-4. Fifth, H.264 outperformed other tested codecs for synthetic video compression. The results of our research can provide common rules for online gaming compression over relatively low bitrates, as well as guidelines for compressing other synthetic videos such as virtual reality video segments. 
The International Journal of Multimedia \& Its Applications (IJMA) Vol.6, No.2, April 2014

\section{REFERENCES}

[1] Deng, C., W. Lin, B.-S. Lee, C. T. Lau \& M. Paul, (2010) "Comparison between H.264/AVC and Motion jpeg2000 for super-high definition video coding," in the 17th IEEE International Conference on Image Processing (ICIP), pp. 2037-2040.

[2] Wiegand, T., H. Schwarz, A. Joch, F. Kossentini \& G. J. Sullivan, (2003) "Rate-constrained coder control and comparison of video coding standards," IEEE Transactions on Circuits and Systems for Video Technology, Vol. 13, pp. 688-703.

[3] Brown, M., D. Bushmitch, K. Kerpez, D. Waring \& Y. Wang, (2009) "Low-bit rate video codec parameter evaluation and optimization," in Military Communications Conference, MILCOM 2009, IEEE, pp. 1-20.

[4] Zhai, G., J. Cai, W. Lin, X. Yang \& W. Zhang, (2008) "Cross-Dimensional Perceptual Quality Assessment for Low Bit-Rate Videos,” IEEE Transactions on Multimedia, Vol. 10, pp. 1316-1324.

[5] McCarthy, J. D., M. A. Sasse \& D. Miras, (2004) "Sharp or smooth?: comparing the effects of quantization vs. frame rate for streamed video," in Conference on Human Factors in Computing Systems, Vienna, Austria.

[6] Bhat, A., S. Kannangara, Y. Zhao \& I. Richardson, (2012) “A Full Reference Quality Metric for Compressed Video Based on Mean Squared Error and Video Content," IEEE Transactions on Circuits and Systems for Video Technology, Vol. 22.

[7] Winkler, S. \& P. Mohandas, (2008) "The Evolution of Video Quality Measurement: From PSNR to Hybrid Metrics," IEEE Transactions on Broadcasting, Vol. 54, pp. 660-668.

[8] Seshadrinathan, K., R. Soundararajan, A. C. Bovik \& L. K. Cormack, (2010) "Study of Subjective and Objective Quality Assessment of Video,” IEEE Transactions on Image Processing, Vol. 19, pp. 1427-1441.

[9] Huynh-Thu, Q., M.-N. Garcia, F. Speranza, P. Corriveau \& A. Raake, (2011) "Study of Rating Scales for Subjective Quality Assessment of High-Definition Video,” IEEE Transactions on Broadcasting, Vol. 57, pp. 1-14.

[10] Dembla, D., B. Patel, A. Kumar \& Y. Bhomia, (2013) "Comparison of H.264 and MPEG-4 Codec Based on PSNR - Peak Signal to Noise Ratio Algorithm", International Journal of Advanced Research in Computer Science and Software Engineering, Vol. 3, Issue 3, pp. 365-370.

[11] http://www.fraps.com, Accessed 5/12/2013.

[12] http://www.netindex.com, Accessed 5/12/2013.

[13] http://media.ofcom.org.uk, Accessed 5/12/2013.

[14] http://www.ispreview.co.uk, Accessed 5/12/2013.

[15] http://www.ffmpeg.org, Accessed 5/12/2013.

[16] International Telecommunication Union, (2002) "Methodology for the Subjective Assessment of the Quality of Television Pictures,” Recommendation ITU-R BT. 500-11 ITU.

[17] Snedecor, G.W. \& W.G. Cochran (1989) Statistical Methods, 8th ed., Iowa State University, USA. 
The International Journal of Multimedia \& Its Applications (IJMA) Vol.6, No.2, April 2014

\section{AUTHORS}

Dr. Ayman M. Abdalla has been a member of the Faculty of Science and Information Technology at Al-Zaytoonah University since 2001, where he held different positions including the Chair of the Department of Multimedia Systems. He received his Ph.D. in computer science from the University of Central Florida, FL, USA; and his Master's and Bachelor's degrees in computer science from Montclair State University, NJ, USA. He has experience in research and teaching in the United States and Jordan in addition to working in software development in a company in the United States.

Dr. Ahmad A. Mazhar has been a member of the Faculty of Science and Information Technology at Al-Zaytoonah University since 2008. He received his Ph.D. in 2013 from De Montfort University, UK; his Master's degree in computer science from Al- Balqa' Applied University, Salt, Jordan; and his Bachelor's degree in computer science from Al-Zaytoonah University, Amman, Jordan.

Mr. Mosa Salah has been a member of the Faculty of Science and Information Technology at Al-Zaytoonah University since 2008. He received his Master's degree in computer science from the Arab Academy for Finance and Banking, Amman, Jordan; and his Bachelor's degree in computer science from Al-Zaytoonah University, Amman, Jordan.
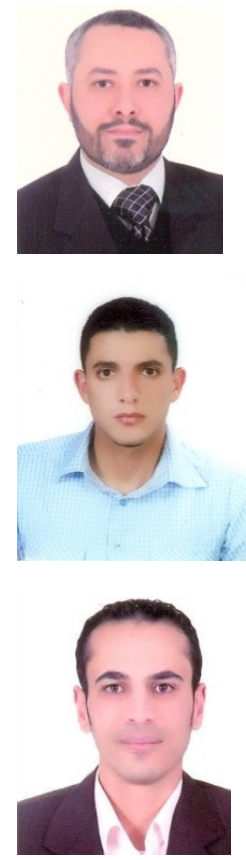

Mrs. Sahar A. Khalaf received her Bachelor's degree in computer graphics from Princess Sumaya University for Technology, Amman, Jordan, in 2010 and started working as a lab assistant at Al-Zaytoonah University in 2011. Currently she is an associate researcher working on a major funded project at AlZaytoonah University. 